DOI: https://doi.org/10.11144/Javeriana.umed59-4.neum

\title{
Neumonía adquirida en la comunidad: una revisión narrativa
}

\section{Community-Acquired Pneumonia: A Review of the Literature}

\author{
SAMuel Martínez-Vernaza \\ Pontificia Universidad Javeriana, Colombia \\ Estefanía MCKINLEY \\ Pontificia Universidad Javeriana, Colombia \\ María Juliana Soto \\ Pontificia Universidad Javeriana, Colombia \\ Sandra Gualtero
}

Hospital Universitario San Ignacio, Colombia

a Correspondencia:
martinez.samuel@javeriana.edu.co

Cómo citar: Martínez-Vernaza S, Mckinley E, Soto MJ, Gualtero S. Neumonía adquirida en la comunidad: una revisión narrativa. Univ. Med. 2018;59(4). doi: https://doi.org/10.11144/Javeriana.umed59-4. neum

\section{RESUMEN}

La neumonía adquirida en la comunidad (NAC) continúa siendo una de las principales causas de mortalidad en Colombia, pues es responsable de 13 de cada 100.000 muertes. Su principal agente etiológico es el Streptococcus pneumoniae, seguido por Haemophilus influenzae y Staphylococcus aureus. Se han identificado algunos factores de riesgo como comorbilidades y factores de exposición epidemiológica, los cuales incrementan la posibilidad de contraer una infección por microrganismos específicos. Su diagnóstico debe basarse tanto en la clínica como en hallazgos paraclínicos e imagenológicos. Herramientas como las escalas CURB-65 y PSI asociadas al criterio clínico permiten calcular el riesgo de mortalidad y el área de atención del paciente según su clasificación. En esta revisión se consideran los diferentes elementos para una adecuada evaluación y manejo del paciente que cursa con NAC, el uso de algunos biomarcadores, situaciones especiales para apreciar, como la neumonía severa, y estrategias para una adecuada prevención.

\section{Palabras clave}

neumonía; neumonía bacteriana; tratamiento; biomarcadores; factores de riesgo; índices de severidad de enfermedad.

\footnotetext{
ABSTRACT

Community acquired pneumonia (CAP) is one of the first mortality causes in Colombia it accounts for 13 of every 100,000 deaths per year. Its principal etiologic agent is still Streptococcus pneumoniae, followed by Haemophilus influenzae and Staphylococcus aureus. Several risk factors have been described for CAP and specific pathoghens, such as coomorbidities and exposition factors. Diagnosis is made by clinical findings associated to laboratory workup and radiological evidence. CURB-65 and PSI are the most known and used tools that, in association with clinical evaluation, calculate the mortality risk and evaluate the setting of management. This literature review aims to consider crucial aspects for the correct assessment of CAP patients, biomarkers used in CAP, particular situations such as severe CAP as well as prevention strategies.

Keywords

pneumonia; bacterial pneumonia; biomarkers; severity of illness index; risk factors; treatment.
} 


\section{Introducción}

La neumonía es un proceso infeccioso e inflamatorio del parénquima pulmonar causado por microrganismos, el cual afecta la porción distal de las vías respiratorias y, en ocasiones, involucra el intersticio alveolar (1). Dicho proceso genera un infiltrado celular inflamatorio del espacio alveolar denominado consolidación, que altera el intercambio gaseoso. Para considerar una neumonía como una neumonía adquirida en la comunidad (NAC), el paciente debe presentar el inicio de signos y síntomas al no estar hospitalizado o en las primeras 48 horas de ingreso (1).

\section{Fisiopatología}

El sistema de defensa de la vía aérea es muy efectivo, ya que existen barreras mecánicas y anatómicas, así como inmunidad celular y humoral, que la mantiene estéril $(1,2,3)$. El mecanismo fisiopatológico de la NAC comienza cuando el sistema de defensa pulmonar es sobrepasado por microrganismos patógenos, los cuales se depositan en la superficie alveolar (2). Factores genéticos, como deficiencias particulares del sistema de defensa, o medioambientales, como el tabaquismo y el alcoholismo, pueden deteriorar la funcionalidad del sistema y facilitar la aparición de una neumonía $(3,4)$.

\section{Epidemiología}

Mundialmente, la NAC es la sexta causa de mortalidad general y la primera causa por enfermedades infecciosas. Al año, su incidencia se encuentra alrededor de 1 y 11 por cada 1000 habitantes; de ellos, el 20-42\% requiere hospitalización, y el 10-30\%, manejo en la unidad de cuidados intensivos (UCI). La mortalidad por NAC ambulatoria es del $1-5 \%$, y por NAC hospitalaria es del 5,7-25\%, ascendiendo hasta un $50 \%$ para pacientes en UCI $(5,6,7)$. Según la Asociación Colombiana de Infectología, en
Colombia, la mortalidad por NAC es de 13 por cada 100.000 habitantes al año y es responsable del $4 \%$ de egresos hospitalarios (70.000 en todos los grupos etarios al año). En estudios nacionales se ha establecido que la mortalidad por Streptococcus pneumoniae es del 3\%, y por gérmenes atípicos, del 11,5\%. En mayores de 65 años, la mortalidad es del 19\% y en la población general, la mortalidad por neumonía severa es del $33 \%$ (1).

\section{Etiología}

En los estudios con mejores métodos diagnósticos y de recolección se ha logrado identificar el germen causal solo en el 40-60\% de los casos, en los cuales el más frecuente es Streptococcus pneumoniae, en un 20-60\%; seguido por Haemophilus influenzae (3-10\%); Staphylococcus aureus, bacilos entéricos gramnegativos, Mycoplasma pneumoniae, Clamydophila pneumoniae, Legionella pneumophila y virus respiratorios $(7,8,9)$. Existen ciertos factores de riesgo y condiciones que incrementan la probabilidad de infección con algunos microrganismos específicos, mencionados en la tabla 1 . 
Tabla 1

Factores de riesgo para microrganismos específicos

\begin{tabular}{|l|l|}
\hline \multicolumn{1}{|c|}{ Microrganismo } & \multicolumn{1}{c|}{ Factores de riesgo } \\
\hline $\begin{array}{l}\text { S. pneumoniae } \\
\text { penicilino-resistente }\end{array}$ & $\begin{array}{l}\text { Mayores de 65 años de edad, uso de betalactámicos en los } \\
\text { últimos 3 meses, alcoholismo, enfermedades } \\
\text { concomitantes, inmunosupresión y exposición a menores } \\
\text { en un centro de cuidado diario u hogar infantil. }\end{array}$ \\
\hline $\begin{array}{l}\text { Bacilos entéricos } \\
\text { gramnegativos } \\
\text { (Enterobacteriaceae) }\end{array}$ & $\begin{array}{l}\text { Residencia en un hogar de cuidado crónico o geriátrico, } \\
\text { enfermedad cardiopulmonar, uso reciente de antibióticos y } \\
\text { antecedente de disfagia o aspiración. }\end{array}$ \\
\hline P. aeruginosa & $\begin{array}{l}\text { Enfermedad pulmonar de base (bronquiectasias o } \\
\text { enfermedad pulmonar obstructiva crónica), terapia con } \\
\text { corticosteroides (más de 10 mg de prednisolona/dia), } \\
\text { terapia con antibióticos de amplio espectro por 7 o más dias } \\
\text { en el último mes, malnutrición. }\end{array}$ \\
\hline S. aureus & $\begin{array}{l}\text { Hemodiálisis, uso de drogas intravenosas, infección previa } \\
\text { por influenza, uso de antibióticos previos } \\
\text { (fluoroquinolonas), neumonia necrosante o infección de } \\
\text { piel grave y concomitante. }\end{array}$ \\
\hline L. pneumophila & $\begin{array}{l}\text { Tabaquismo, jóvenes sin enfermedades concomitantes, } \\
\text { sintomas digestivos y neurológicos concomitantes, NAC } \\
\text { grave con afectación multiorgánica. }\end{array}$ \\
\hline Anaerobios & Antecedente de disfagia o aspiración. \\
\hline
\end{tabular}

Fuente: tomado y ajustado de Infectio. 2015;19(1):10-7.

Neumonía por Staphylococcus aureus resistente a meticilina adquirido en la comunidad (SAMR-AC)

Últimamente ha aumentado la presencia del SAMR-AC como causante de neumonía en Colombia, sobre todo desde su surgimiento en infecciones de piel y tejidos blandos (1). Se ha descrito que el cuadro clínico se presenta en jóvenes, previamente sanos, precedido por un cuadro de influenza dado por sintomatología respiratoria severa en el $75 \%$ de los casos, como hemoptisis, fiebre, hipotensión y requerimiento de intubación; al igual que hallazgos paraclínicos como leucopenia, niveles de proteína $\mathrm{C}$ reactiva $>400 \mathrm{~g} / \mathrm{L}, \quad$ y radiológicos como infiltrados alveolares multilobares y cavitaciones $(10,11)$. Es importante el diagnóstico rápido y certero de neumonía por SARM-AC, dado el alto riesgo y mortalidad que genera la enfermedad. También es fundamental el inicio adecuado del tratamiento de manera empírica, pues se ha visto que la mortalidad disminuye de un $60,8 \%$ a un $33,3 \%$, especialmente en pacientes que ingresan en choque séptico (10).

\section{Neumonía por Streptococcus pneumoniae resistente a penicilina}

Por medio del Sistema de Redes de Vigilancia de los Agentes Responsables de Neumonías y Meningitis Bacterianas de la Organización Panamericana de la Salud, se obtuvo una serie de aislamientos de Streptococcus pneumoniae entre 2006 y 2015. Los perfiles de susceptibilidad mostraron una sensibilidad promedio a la penicilina del $92,34 \%$ en cuadros de no meningitis, es decir, un $7,6 \%$ de aislamientos eran resistentes a la penicilina, lo cual debe tenerse en cuenta en cuadros de neumonía que no respondan al tratamiento empírico (12). En cuanto a otros antimicrobianos, se encontró que la tasa de sensibilidad a ceftriaxona es del $94 \%$, de resistencia a trimetoprima-sulfametoxazol es cerca del $20 \%$ y de sensibilidad de vancomicina, cloranfenicol y eritromicina es mayor del $90 \%$ (12).

\section{Neumonía por Mycobacterium tuberculosis}

En Colombia, Mycobacterium tuberculosis es uno de los agentes causantes de NAC, con un 2,9\% de los aislamientos según Vélez et al. (7). Es pertinente tener en cuenta este agente como posible causante de NAC en un país endémico como el nuestro. $\mathrm{Su}$ presentación puede estar dada por tos, disnea, dolor torácico, fiebre, síntomas constitucionales (pérdida de peso y malestar general), además de hallazgos imagenológicos como consolidaciones, cavitaciones o derrame pleural en lóbulos inferiores, língula y lóbulo medio (13). El diagnóstico requiere la sospecha clínica para establecer métodos diagnósticos que identifiquen a tiempo esta patología y así lograr un tratamiento adecuado y oportuno, evitando la prolongación de la enfermedad y complicaciones mayores en los pacientes $(13,14)$. 


\section{Diagnóstico de la neumonía adquirida en la comunidad}

El diagnóstico se realiza a partir de los datos obtenidos durante la anamnesis y el examen físico, así como interpretación de imágenes y exámenes paraclínicos (1). Los siguientes criterios ayudan a un diagnóstico adecuado:

1. Al menos un signo, como dificultad respiratoria, matidez, estertores, egofonía, frémito vocal aumentado y aumento de las vibraciones vocales a la palpación. Síntomas como fiebre, malestar general, tos con expectoración, disnea, etc.

2. Signos de respuesta inflamatoria sistémica, como fiebre $>38{ }^{\circ} \mathrm{C}$ o hipotermia $<36{ }^{\circ} \mathrm{C}$, frecuencia cardiaca $>90$ l. p. m., frecuencia respiratoria $>$ 30 r. p. m., leucocitosis $<12.000$ cel./ $\mathrm{mm} 3$ o leucopenia $<4000 \mathrm{cel} . / \mathrm{mm}^{3}$, o presencia de cayados de $>10 \%$.

3. Radiografía de tórax con infiltrados alveolares, intersticiales segmentarios o de cualquier tipo en más de un lóbulo, o derrame pleural.

4. Ausencia de cualquier otra enfermedad que explique los síntomas.

5. Considerar neumonía por aspiración en pacientes cuya aspiración fue presenciada, alteración del estado de conciencia, trastornos de la deglución, obstrucción intestinal o alteración del reflejo nauseoso, o con hallazgos en la radiografía de tórax en lóbulos inferiores (aspiración sentado), segmentos superiores de lóbulos inferiores o posteriores de lóbulos superiores (aspiración en decúbito).

\section{Diagnósticos diferenciales de la neumonía adquirida en la comunidad}

Se debe diferenciar de bronquitis aguda, exacerbación aguda de enfermedad pulmonar obstructiva crónica, edema pulmonar, tromboembolismo pulmonar, neumonitis por radiación o medicamentos y hemorragia alveolar (15).

\section{Clasificación}

La NAC se clasifica de acuerdo con puntajes o escalas que evalúan diversas características clínicas y paraclínicas. El objetivo general de las escalas se centra en la predicción de mortalidad a 30 días, severidad de la enfermedad y decisión en el lugar de hospitalización del paciente con NAC (4).

Tanto el CURB-65, que evalúa las variables confusión, urea, frecuencia respiratoria, presión arterial y edad $>65$ años (16), como el Pneumonia Severity Index, que evalúa datos demográficos, enfermedades concomitantes, hallazgos en el examen físico y hallazgos en ayudas diagnósticas (17), se consideran herramientas fuertes para usar como predictores de severidad y mortalidad $(4,18)$. Se ha demostrado en los últimos años que marcadores clínicos complementarios a estas escalas mejoran el rendimiento de la predicción de mortalidad a 30 días $(19,20,21)$. Además, es muy importante tener en cuenta que los puntajes descritos deben asociarse al criterio clínico y a factores sociales y económicos del paciente (4).

\section{Neumonía severa adquirida en la comunidad}

Aproximadamente, el 10\% de los pacientes con NAC desarrollará una neumonía severa, la cual en la mayoría de los casos requerirá manejo en la UCI, debido a la presencia de choque con necesidad de vasopresores o de insuficiencia respiratoria, que necesita ventilación mecánica. Los pacientes con mayor riesgo de presentar una NAC severa son aquellos con edad avanzada, presencia de comorbilidades, residencia en hogar geriátrico, inmunosupresión o alteración del estado mental. Detectar a estos pacientes es esencial, porque son ellos los que tienen una tasa de mortalidad más alta y requieren una atención con más apoyo. Sin embargo, se reitera 
que aproximadamente un tercio de los pacientes con neumonía severa eran previamente sanos (2). Para la clasificación de neumonía severa existen una serie de criterios establecidos por la Sociedad de Enfermedades Infecciosas de América y la Sociedad Torácica Americana, en 2007, los cuales permiten priorizar el manejo en la UCI o en una unidad de cuidados intermedios (5). Estos se pueden ver detalladamente en la tabla 2 (5).

\section{Tabla 2}

Criterios para neumonía severa según la Sociedad de Enfermedades Infecciosas de América y la Sociedad Torácica Americana

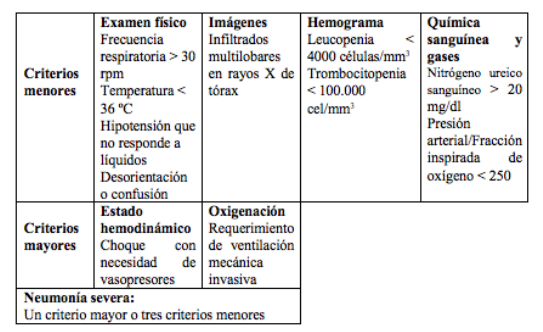

\section{Exámenes paraclínicos en la neumonía adquirida en la comunidad}

Todos los pacientes con sospecha de NAC deben tener radiografía de tórax y pulsoximetría. Exámenes básicos como función renal, hepática y electrolitos resultan de gran ayuda para la clasificación de acuerdo con las diferentes escalas disponibles (3); sin embargo, la disponibilidad de ayudas diagnósticas para NAC es muy amplia y en algunos casos costosa, por tanto, algunos paraclínicos se reservan para pacientes hospitalizados o con alto riesgo de complicaciones.

La búsqueda etiológica en pacientes ambulatorios es opcional, dado que usualmente la respuesta al tratamiento empírico es adecuada; por ende, se recomienda solo cuando se sospecha infección por microrganismos con implicaciones epidemiológicas o en el tratamiento, por ejemplo, de tuberculosis, influenza H1N1, microrganismo con resistencia antimicrobiana, bioterrorismo o falla al tratamiento ambulatorio.

En pacientes que requieren manejo intrahospitalario, se sugiere solicitar hemograma, función renal y glucemia. Algunos pacientes pueden tener indicaciones de otros exámenes como gases arteriales, Gram y cultivo de esputo, baciloscopia seriada de esputo y panel viral según recomendaciones epidemiológicas. En sospecha de un germen atípico (no mejoría a las 72 horas) se recomienda una tomografía computarizada y fibrobroncoscopia. Igualmente, se plantea toma de hemocultivos en pacientes que requieren hospitalización en la UCI, pacientes con abuso de alcohol, asplenia, leucopenia, derrame pleural, neumonía necrosante o influenza previa. Si se encuentra con un derrame pleural en estudio $>$ $1 \mathrm{~cm}$ proyección en decúbito lateral, $>5 \mathrm{~cm}$ en proyección lateral de pie o por ecografía de tórax, se debe realizar una toracentesis y enviar líquido para pruebas bioquímicas y microbiológicas con muestra de sangre comparativa correspondiente (1).

Si el paciente presenta disfunción orgánica, se recomienda solicitar tiempos de coagulación, gases arteriales, bilirrubinas, lactato y gases venosos centrales para orientar la terapia de reanimación temprana (1). Además, se solicita cultivo de esputo, secreción orotraqueal o fibrobroncoscopia, y se evalúa la necesidad de pruebas pareadas de suero para gérmenes atípicos junto con antígeno urinario para Legionella spp. y S. pneumoniae o sospecha de C. burnetti, virus, C. psittaci y P. jirovecci (1).

\section{Biomarcadores incluidos en la práctica clínica}

Usualmente, la evaluación de infecciones pulmonares se da con marcadores inflamatorios, como el recuento leucocitario y los niveles de proteína $\mathrm{C}$ reactiva; sin embargo, ha venido en aumento el uso de los niveles séricos de la procalcitonina (22), cuyo incremento en concentración sanguínea se ha visto asociado a infecciones bacterianas, y sus niveles se ven disminuidos en infecciones virales (23), por lo que actualmente se utiliza como uno de los biomarcadores para el diagnóstico, el pronóstico y el tratamiento de las NAC. Se ha documentado que los niveles séricos de procalcitonina se ven elevados en neumonías bacterianas y choque 
séptico (24). Por tanto, no se recomienda usar antibióticos en pacientes con niveles de procalcitonina $<0,25 \mu \mathrm{g} / \mathrm{L}$, pues se asocian más con etiología viral (24).

Adicionalmente, se ha planteado el uso de la proadrenomedulina, una hormona peptídica producida en situaciones de inflamación sistémica en múltiples tejidos para generar vasodilatación y como agente inmunomodulador y bactericida $(25,26)$, pues se ha encontrado que su medición de niveles séricos tiene una validación tan específica como una serie de scores de severidad, como el Sepsis-Related Organ Failure Assesment y el Pneumonia Severity Index, incluso con mejor rendimiento que otros biomarcadores como la procalcitonina (26). Sus concentraciones normales se encuentran menores de 0,52 nmol/L y se recomienda su medición $12 \mathrm{~h}$ después del inicio de la primera dosis de antibióticos y 48 h después, la cual si se encuentra elevada indica alta mortalidad a corto y largo plazo (26). Es un biomarcador de nueva instauración en la práctica mundial, útil para evaluar mortalidad a corto y largo plazo con alta especificidad (27).

\section{Tratamiento de la neumonía adquirida en la comunidad}

El tratamiento inicial de la NAC es empírico, pues no existe una herramienta diagnóstica con una sensibilidad y especificidad del 100\%. Se debe realizar teniendo en cuenta la epidemiología, los perfiles locales de resistencia microbiana, la disponibilidad local y los costos (5).

Medidas recomendadas en todos los pacientes $(1,5)$. Suplencia de oxígeno para lograr una saturación mayor del 90\%, hidratación y balance electrolítico, profilaxis para eventos tromboembólicos, terapia respiratoria, manejo de enfermedades concomitantes, monitorización hemodinámica y respiratoria no invasiva para pacientes que se encuentren en la UCI, más reanimación protocolizada (hipoperfusión tisular inducida por sepsis).
Manejo empírico según los grupos del CURB-65 y criterios de severidad la Sociedad de Enfermedades Infecciosas de América y la Sociedad Torácica Americana (esquema de manejo en la tabla 3) (5). Deben iniciar en la primera hora en pacientes con sepsis o choque séptico; en los demás, en las primeras 4-6 h $(1,5)$.

Tabla 3

Tratamiento según grupos de pacientes basados en CURB-65

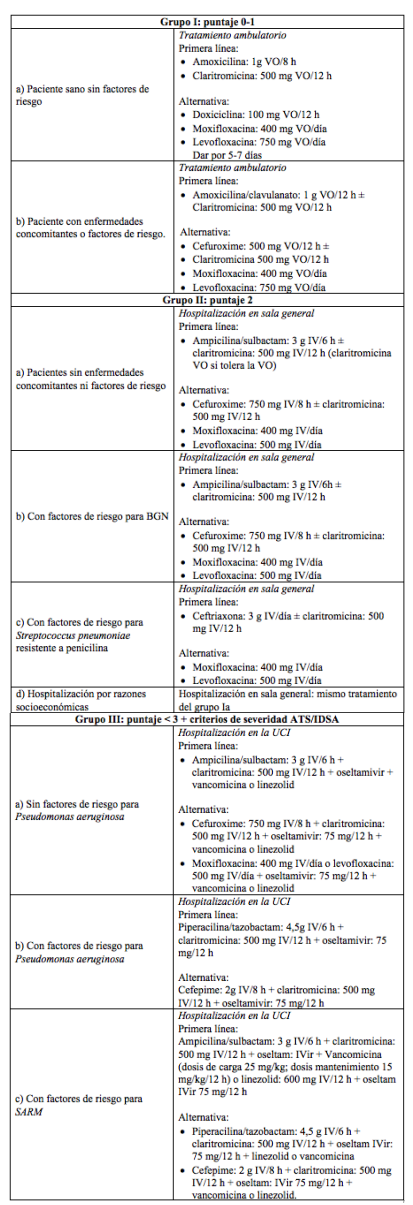

Manejo dirigido. Las opciones terapéuticas se pueden simplificar si el agente etiológico está establecido o se sospecha fuertemente $(1,2)$. Las pruebas que identifican los gérmenes pueden demorarse hasta $72 \mathrm{~h}$. En este contexto, se debe desescalar la terapia empírica inicial si la susceptibilidad es de un menor espectro y así ayudar en la problemática de la resistencia microbiana (5). 


\section{Macrólidos en la neumonía adquirida en la comunidad}

El uso de macrólidos se ha convertido en una herramienta terapéutica de gran impacto para la $\mathrm{NAC}$, en especial cuando se quiere garantizar el cubrimiento de gérmenes atípicos y en cuadros de NAC severa $(1,5)$. Si bien es cierto que el uso de la terapia combinada de macrólidos y betalactámicos en NAC ha mostrado efectividad en los desenlaces de mortalidad $(5,25)$, algunos datos de estudios principalmente observacionales muestran que el beneficio se da únicamente en los pacientes cursando con NAC severa (28).

\section{Corticoesteroides en la neumonía adquirida en la comunidad}

La terapia con corticoesteroides se ha venido manejando como una opción importante al enfrentarse a los pacientes con NAC. Sus propiedades radican principalmente en la inhibición de la expresión de citocinas proinflamatorias, que están en relación directa con la respuesta inmune generada en NAC (22). Esto puede resultar beneficioso en algunos casos; sin embargo, lo encontrado por Wan et al. (28) en una revisión sistemática y metaanálisis muestra que el uso de corticoesteroides en NAC no tuvo un efecto estadísticamente significativo para el desenlace de mortalidad. Sí se encontró una reducción en el riesgo de presentar síndrome de dificultad respiratoria aguda en estos pacientes (28).

\section{Prevención de la neumonía adquirida en la comunidad}

Según las Guías para la inmunización del adolescente y adulto, de la Asociación Colombiana de Infectología (29), para la prevención de la infección por S. pneumoniae se tienen en cuenta dos vacunas: la antineumocócica de polisacáridos (PPSV23), con los 23 serotipos más frecuentes, y la conjugada multivalente (PCV13), que contiene 7, 10 o 13 polisacáridos unidos a una proteína transportadora que induce inmunidad celular dependiente de células T (29).

Se recomienda que los adultos de 19 a 59 años en condiciones de inmunocompromiso o comorbilidades, o mayores de 60 años sanos, deben ser inmunizados con PCV13 y PPSV23 para prevenir la enfermedad invasiva y neumonía por S. pneumoniæ. Igualmente, todo adulto que ha recibido PCV13 por inmunocompromiso debería recibir una dosis de PPSV23 a las 8 semanas después de su aplicación, con repetición en única dosis de PPSV23 en 5 años.

Por otro lado, se encuentra la vacuna contra el virus de la influenza, indicada para aplicación anual en población cautiva (hogares geriátricos, comedores comunitarios, etc.) al igual que en mayores de 60 años (29). Adicionalmente, la inmunización contra influenza del personal de salud y cuidador de la persona mayor podría disminuir la infección en el anciano, y viceversa (29).

\section{Conclusiones}

La NAC continúa siendo una de las principales causas de mortalidad en Colombia y en el mundo. Su diagnóstico debe basarse en una adecuada anamnesis y examen físico junto con el uso de imágenes diagnósticas y paraclínicos. Se ha planteado el uso de biomarcadores como la procalcitonina y la proadrenomedulina para complementar el estudio de los pacientes cursando con NAC.

El principal agente etiológico de NAC continúa siendo el S. pneumoniae; sin embargo, es necesario evaluar diferentes factores de riesgo que permitan sospechar la infección por virus respiratorios, bacterias atípicas, hongos, parásitos y microrganismos emergentes como $P$. aeruginosa, SAMR-AC y M. tuberculosis.

Posterior al diagnóstico, se pueden utilizar herramientas como el CURB-65 y el Pneumonia Severity Index en conjunto con el criterio clínico y la situación socioeconómica del paciente, para evaluar la mortalidad y establecer el escenario para el manejo. Igualmente, es de gran importancia identificar los pacientes que 
cursen con neumonía severa por el alto riesgo de mortalidad que requieren manejo en la UCI o en una unidad de cuidados intermedios.

El tratamiento de la NAC se fundamenta en la implementación de medidas básicas junto con el manejo antimicrobiano empírico o, en caso de identificar el germen causal, un manejo dirigido. Se recomienda continuar con el uso de macrólidos junto con betalactámicos en NAC severa. No se recomienda el uso de corticoesteroides para NAC, pues no se cuenta con evidencia que demuestre mejores desenlaces en mortalidad. Se deben aplicar las diferentes estrategias de prevención disponibles para microorganismos causantes de NAC, como vacunación para S. pneumoniae y para influenza.

\section{Agradecimientos}

A la Dra. Alejandra Cañas Arboleda, por su valiosa colaboración durante el desarrollo de la revisión.

\section{Referencias}

1. Asociación Colombiana de Neumología y Cirugía de Tórax, Asociación Colombiana de Infectología, Asociación Colombiana de Medicina Interna. Recomendaciones para el diagnóstico, tratamiento y prevención de la neumonía adquirida en la comunidad en adultos. Infectio [internet]. 2004;8(1):8-44. Disponible en: http://www.revistainfectio.org/inde x.php/infectio/article/view/438/443

2. Bennett J, Dolin R, Blaser M. Mandell, Douglas, and Bennett's principles and practice of infectious diseases. Principles and Practice of Infectious Diseases. Philadelphia: Elsevier; 2014.

3. Arshad H, Fasanya A, Cheema

T, Singh AC. Acute pneumonia.
Crit Care Nurs Q. 2016 AprJun;39(2):148-60.

4. Menéndez R, Torres A, Aspa J, Capelastegui A, Prat C, Rodríguez de Castro F. Community-acquired pneumonia: New guidelines of the Spanish Society of Pulmonology and Thoracic Surgery (SEPAR). Arch Bronconeumol. 2010;46(10):543-58.

5. Mandell LA, Wunderink RG, Anzueto A, Bartlett JG, Campbell GD, Dean NC, et al. Infectious Diseases Society of America/American Thoracic Society consensus guidelines on the management of communityacquired pneumonia in adults. Clin Infect Dis. 2007;44 Suppl 2(1537-6591):S27-72.

6. Isturiz RE, Luna CM, Ramírez J. Clinical and economic burden of pneumonia among adults in Latin America. Int J Infect Dis. 2010 Oct;14(10):e852-6.

7. Vélez L, Rueda Z, Aguilar Y, Ortega $\mathrm{H}$, Montufar FE, Arroyave $\mathrm{M}$, et al. Caracterización clínica y etiológica de NAC, Valle de Aburrá. Infectio. 2006; 10:103

8. Caballero A, Polanía E, Gordillo M, Martínez O, Torrado E, et al. Agentes etiológicos de Neumonía Adquirida en Comunidad (NAC) en pacientes adultos inmunocompetentes que consultan al servicio de urgencias de la Clínica Reina Sofía, Bogotá, 2006-2007. Rev Médica Sanitas. 2010;13:8-18.

9. Robledo J, Sierra P, Bedoya F, Londoño A, Porras A, Luján $M$, et al. Neumonías adquiridas en la comunidad en adultos: un estudio etiológico prospectivo con énfasis en el diagnóstico. Rev Colomb Neumol. 2003;15:7-14.

10. Rubinstein E, Kollef MH, Nathwani D. Pneumonia caused by methicillin- 
resistant Staphylococcus aureus. Clin Infect Dis. 2008;46(S5):S378-85.

11. Obed M, García-Vidal C, Pessacq P, Mykietiuk A, Viasus D, Cazzola L, et al. Características clínicas y pronóstico de la neumonía adquirida en la comunidad causada por Staphylococcus aureus resistente a la meticilina. Enferm Infecc Microbiol Clin. 2014 Jan;32 (1):23-7.

12. Instituto Nacional de SaludGrupo de Microbiología. Informe nacional de SIREVA II Colombia 1994-2005 [internet]. Disponible en: https://www.ins.gov.co/buscador-e ventos/Informacin\%20de\%20laborator io/Informe\%20 Nacional\%20SIREVA \%20II\%20Colombia\%201994-2005\%2 OStreptococcus\%20pneumoniae.pdf

13. Álvarez MF, Ramírez Quintero JD, Mejía SA. Neumonía y neumotórax espontáneo por tuberculosis. Acta Med Colomb [internet]. 2016;41(1):62-6. Disponible en: http://www.scielo.org.c o/pdf/amc/v41n1/v41n1a12.pdf

14. Gutiérrez CJ, Zamudio RS. Neumonía tuberculosa: Reporte de 20 casos y estudio caso-control. Acta Med Peru. 2001;18(1):5-11.

15. Longo D, Fauci A, Kasper D, Hauser S. Harrison principios de medicina interna. New York: McGraw Hill; 2012.

16. Macfarlane J. CURB-65 [Internet]. Disponible en: https://www.mdcalc.co $\mathrm{m} /$ curb-65score-pneumonia-severity

17. Michael J. Fine. PSI/ PORT SCORE [Internet]. Disponible en: https://www.mdcalc.com/psi-port-s core-pneumonia-severity-index-cap\#c reator-insights

18. Alan M, Grolimund E, Kutz A, Christ-Crain M, Thomann R, Falconnier C, et al. Clinical risk scores and blood biomarkers as predictors of long-term outcome in patients with community-acquired pneumonia: a 6 - year prospective follow-up study. J Intern Med. 2015;278(2):174-84.

19. Yeon Lee S, Cha S-I, Seo $\mathrm{H}$, Oh S, Choi K-J, Yoo S-S, et al. Multimarker Prognostication for Hospitalized Patients with Community-acquired Pneumonia. Intern Med. 2016;55(8):887-93.

20. Viasus D, Del Río-Pertuz G, Simonetti AF, García-Vidal C, AcostaReyes J, Garavito A, et al. Biomarkers for predicting short-term mortality in community-acquired pneumonia: A systematic review and meta-analysis. J Infect. 2016;72(3):273-82.

21. Aujesky D, Fine MJ. The pneumonia severity index: a decade after the initial derivation and validation. Clin Infect Dis. 2008;47 Suppl 3:S133-9.

22. Krüger S, Ewig S, Marre R, Papassotiriou J, Richter K, Von Baum $\mathrm{H}$, et al. Procalcitonin predicts patients at low risk of death from community-acquired pneumonia across all CRB-65 classes. Eur Respir J. 2008;31(2):349-55.

23. Gilbert DN. Procalcitonin as a biomarker in respiratory tract infection. Clin Infect Dis. 2011;52 (suppl. 4):346-50.

24. Christ-Crain M, Jaccard-Stolz D, Bingisser R, Gencay MM, Huber PR, Tamm M, et al. Effect of procalcitoninguided treatment on antibiotic use and outcome in lower respiratory tract infections: Cluster-randomised, singleblinded intervention trial. Lancet. 2004;363 (9409):600-7.

25. Leoni D, Rello J. Severe community-acquired pneumonia: optimal management. Curr Opin Infect Dis. 2017;30(2):240-7.

26. Pereira JM, Azevedo A, Basílio C, Mergulhão P, Paiva JA. Midregional proadrenomedullin: An early 
marker of response in critically ill patients with severe communityacquired pneumonia? Rev Port Pneumol. 2016;22(6):308-14. doi: 10.1016/j.rppnen.2016.03.012

27. Julián-Jiménez A, González del Castillo J, Candel Fj. Usefulness and prognostic value of biomarkers in patients with community-acquired pneumonia in the emergency department. Med Clin (Barc). 2017;148(11):501-10. doi: 10.1016/ j.medcli.2017.02.024

28. Wan YD, Sun TW, Liu ZQ, Zhang SG, Wang LX, Kan QC. Efficacy and safety of corticosteroids for community-acquired pneumonia: a systematic review and meta-analysis. Chest. 2016 Jan;149(1):209-19.

29. Manuel J, Mu G, César J, Rincón G, Alí A, Cano CA, et al. Guías para la inmunización del adolescente y adulto en Colombia: Documento de actualización, 2016. Infectio [Internet]. 2016;20(4):192-210. Disponible en: http://www.scielo.org.c o/pdf/inf/v20n4/0123-9392-inf-20-0400192.pdf 